\title{
Using Constant Switching Frequency in a Linear- Assisted DC-DC Switching Regulator
}

\author{
Herminio Martínez-García \\ Barcelona College of Industrial Engineering (EUETIB) \\ Department of Electronics Engineering \\ Technical University of Catalonia - BarcelonaTech (UPC) \\ c/ Comte de Urgell, 187, 08036 - Barcelona (Spain) \\ herminio.martinez@upc.edu
}

\begin{abstract}
This article shows the proposal of a linear-assisted converter or linear- $\&$-switching hybrid converter with a constant switching frequency. The control loop of the system is based on the current-mode technique. The main disadvantage of a converter with current-mode control is the inherent instability of the loop when switch duty ratios are greater than 0.5 . In order to make stable the proposed linear-assisted converter, the article shows the technique based on a slope compensation.
\end{abstract}

Keywords-DC-DC switching converters; voltage linear regulators; linear-assisted $D C-D C$ voltage regulators

\section{INTRODUCTION (HEADING 1)}

As it is well known, series linear regulators have been structures widely used in power supply systems for decades, providing output voltages with low or moderate consumes and currents [1], [2]. This kind of voltage regulators has several advantages that lead their use. However, in spite of these advantages, linear regulators suffer from some serious drawbacks. One significant example is that the efficiency of these structures hardly exceeds the $50 \%$ and the series-pass transistor has to support all the current demanded by the load. Thus, in high power applications, this component has to be dimensioned (both electrically and thermically) in order to dissipate powers with high values. This point increases the price of the supply system significantly. As a result, they are not recommendable in some supply systems, especially for high power.

The alternative is also widely known: $D C-D C$ switching converters [3]-[5]. The advantages of these structures are also evident opposite to linear regulators: the efficiency of them, in spite of not arriving at the $100 \%$ due to the omnipresent circuit losses, is near to this optimal value. However, the design and implementation of this sort of converters is a more complex process than in linear regulators, especially their control loops when both line and load regulations are desired. In addition, the intrinsic switched nature of these converters produces ripples in the output voltage and an increment of the EMIs in neighboring electronic systems.

Last years, a third possibility has been presented: linear$\&$-switching hybrid converters or linear-assisted converters [6], [7]. These converters are compact structures, which make good use of the advantages from previous alternatives (linear regulators and switching converters). With them, some of the aforementioned drawbacks are minimized as, for instance, the low efficiency and the high power dissipation in linear regulators, or the complexity in the design of the control for switching converters.

In general, linear-assisted converters presented in literature are variable-frequency structures [8]-[10]. In this case, the switching frequency depends on the parameters of the converter and is not fixed by an external clock. This drawback affects the design of the converter and the selection of its components. In addition, this feature is not desirable in some applications because variable switching frequency could insert into the bandwidth of the neighboring electronic circuits or even of the electronic system that the converter supplies. Thus, with the design of a constant-frequency linear-assisted converter, it is possible to choose the desired switching frequency independently of the component values.

In this article, a proposal of constant-frequency linearassisted converter, especially suitable as DC-DC regulator for photovoltaic solar-powered facilities, is presented. Section II presents a review of variable-frequency linear-assisted converters, section III and IV show the proposal of the constant-frequency linear-assisted converter, revealing the problem of its stability and the solution adopted to avoid it. Finally, section V concludes with some simulation results that validate the proposed structure.

\section{REVIEW OF VARIABLE-FREQUENCY LINEAR-ASSISTED CONVERTERS}

As we can see in figure 1, the proposed configuration makes use of an analog comparator that controls the conduction or cut of the transistor $Q_{1}$ and fixes the switching frequency. Note that the objective of the switching converter is to provide the excess of current that the linear regulator does not supply. Consequently:

$$
I_{\text {out }}=i_{\text {reg }}(t)+i_{L}(t)
$$

Just not considering in a first approximation any hysteresis in the comparator $C M P_{1}$, the operation of the circuit is just as it is explained as follows: if the load current is below a boundary current value, that we name as threshold switching current, $I_{\gamma}$, the output of the comparator $C M P_{1}$ is held low. Thus, the 
switching converter will be disabled and, therefore, the current through the inductance $L_{1}$ will be zero. As a result, the linear regulator supplies the load $R_{L}$, providing all the output current $\left(I_{\text {reg }}=I_{\text {out }}\right)$.

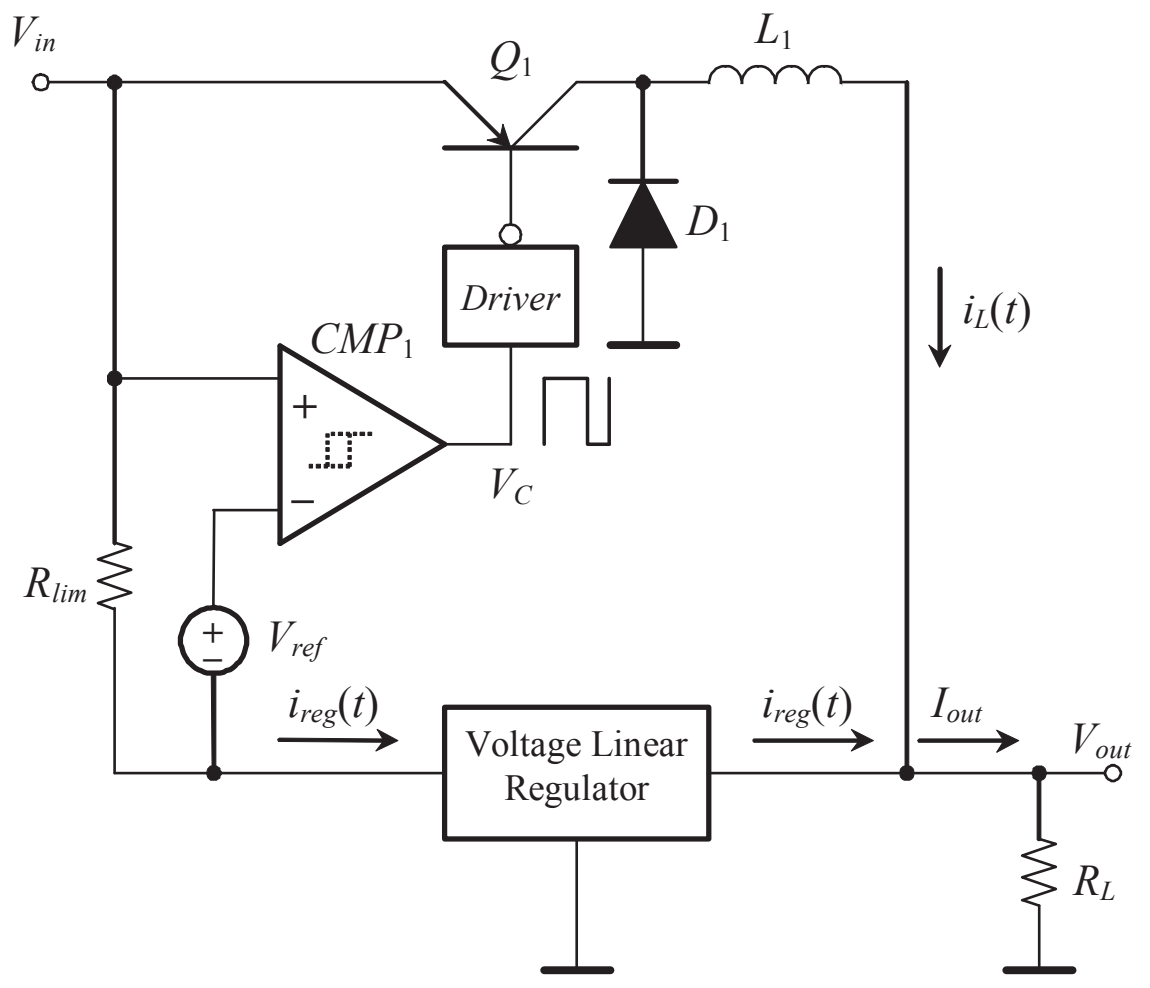

Fig. 1. Basic structure of a self-switched hybrid converter that supplies a load $R_{L}$.

However, in the moment that load current increases and goes slightly beyond this limit current $I_{\gamma}$, the comparator output will pass to high level, increasing the inductance current in a linear form. Taking into account expression (1) and that the load current is constant (equal to $V_{\text {out }} / R_{L}$ ), the regulator current $i_{\text {reg }}(t)$ will tend to decrease also linearly (figure 2 ), just reaching a value below $I_{\gamma}$. In this moment, the comparator changes its output from high to low level, the transistor $Q_{1}$ goes to off and forces the inductance current to decrease. Then, when the current in the inductance decreases to a value in which $i_{\text {reg }}(t)>I_{\gamma}$, the comparator changes from low to high level, and the cycle starts again.

Both reference voltage $V_{\text {ref }}$ and shunt resistor $R_{\text {lim }}$ determine the value of the threshold switching current $I_{\gamma}$. Fixing $I_{\gamma}$, the value of the $R_{\text {lim }}$ can be obtained by expression (2).

$$
R_{\mathrm{lim}}=\frac{V_{r e f}}{I_{\gamma}}
$$

With the objective of not decreasing the efficiency of the converter, the value of the dissipated power by the internal transistor of the linear regulator must be reduced to the utmost. For this reason, the current $I_{\gamma}$ has to be the minimum and necessary value to make the linear regulator work properly, without penalizing its good regulation characteristics.

The delays of the electronic circuits determine a little hysteresis that limits the maximum switching frequency. Nevertheless, with the objective of fixing this maximum frequency to a suitable value (in order not to increase the switching losses significantly), it is convenient to add a hysteresis to the comparator $C M P_{1}$.

The experimental implementation of the self-switched hybrid regulator presented in figure 1 can be found in [9] and [10]. Figure 3 shows the waveforms of a variable-frequency linear-assisted converter with $V_{i n}=10 \mathrm{~V}$. In the same figure, it is observed the response of the system to an input voltage step from $10 V$ to $13 V$ in the time instant $t=20 \mu s$, and a step change of the load resistor of the $50 \%$ (from $10 \Omega$ to $5 \Omega$ ) in $t=40 \mu \mathrm{s}$.

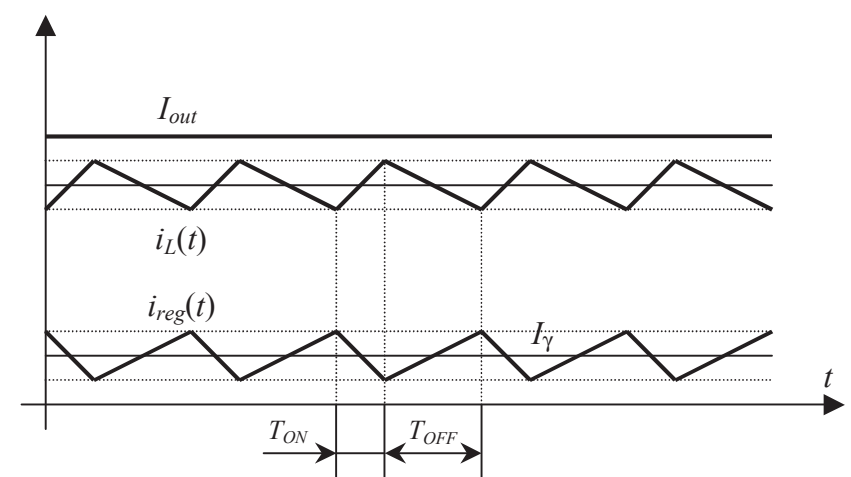

Fig. 2. Currents through the load resistor, inductance $L_{1}$ and linear regulator in the steady state.

The switching frequency is given by (3), being the upper and lower switching threshold levels of the comparator (Schmitt trigger) $V_{H}$ and $V_{L}$, respectively. 


$$
f=\frac{R_{\text {lim }}}{L_{1}} \frac{V_{\text {out }}}{V_{H}-V_{L}}\left(1-\frac{V_{\text {out }}}{V_{\text {in }}}\right)
$$

From equation (3) we obtain the switching frequency is a function of the hysteresis of the comparator, input and output voltages and $R_{\text {lim }}$ and $L_{1}$ values. This dependency from the aforementioned values is an important drawback. Note that the switching frequency depends on the possible disturbances in $V_{\text {in }}$ and tolerances and dispersions in passive components of the converter.
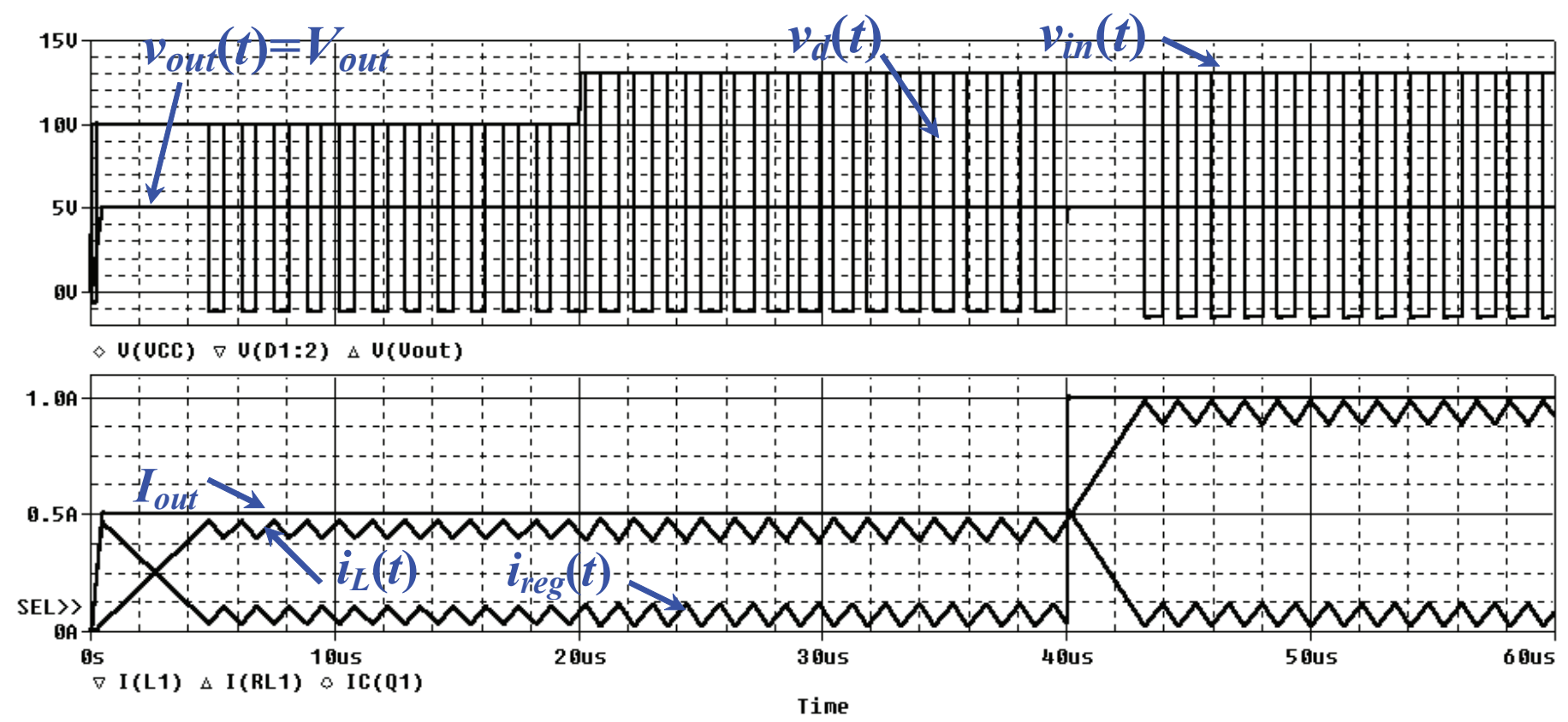

Fig. 3. Transient response of the variable-frequency linear-assisted converter with $V_{i n}=10 \mathrm{~V}$. It can be seen the response of the circuit to an input step from $10 \mathrm{~V}$ to $13 V$ in the time instant $t=20 \mu \mathrm{s}$, and when there is a decrement of the load resistance from $10 \Omega$ to $5 \Omega$ in $t=40 \mu \mathrm{s}$.

\section{CONSTANT-FREQUENCY LINEAR-ASSISTED CONVERTER}

Figure 4 shows a constant-frequency linear-assisted converter. As the variable-frequency converter shown in figure 1, this topology has a switching converter and a linear regulator. However, now the switching frequency is fixed by the external clock signal $V_{C L K}$. This signal is applied to the set input of a RS flip-flop and the output of the comparator is applied to the reset input of the bistable. The output of the flipflop is applied to the base or gate of the switch $Q_{1}$.

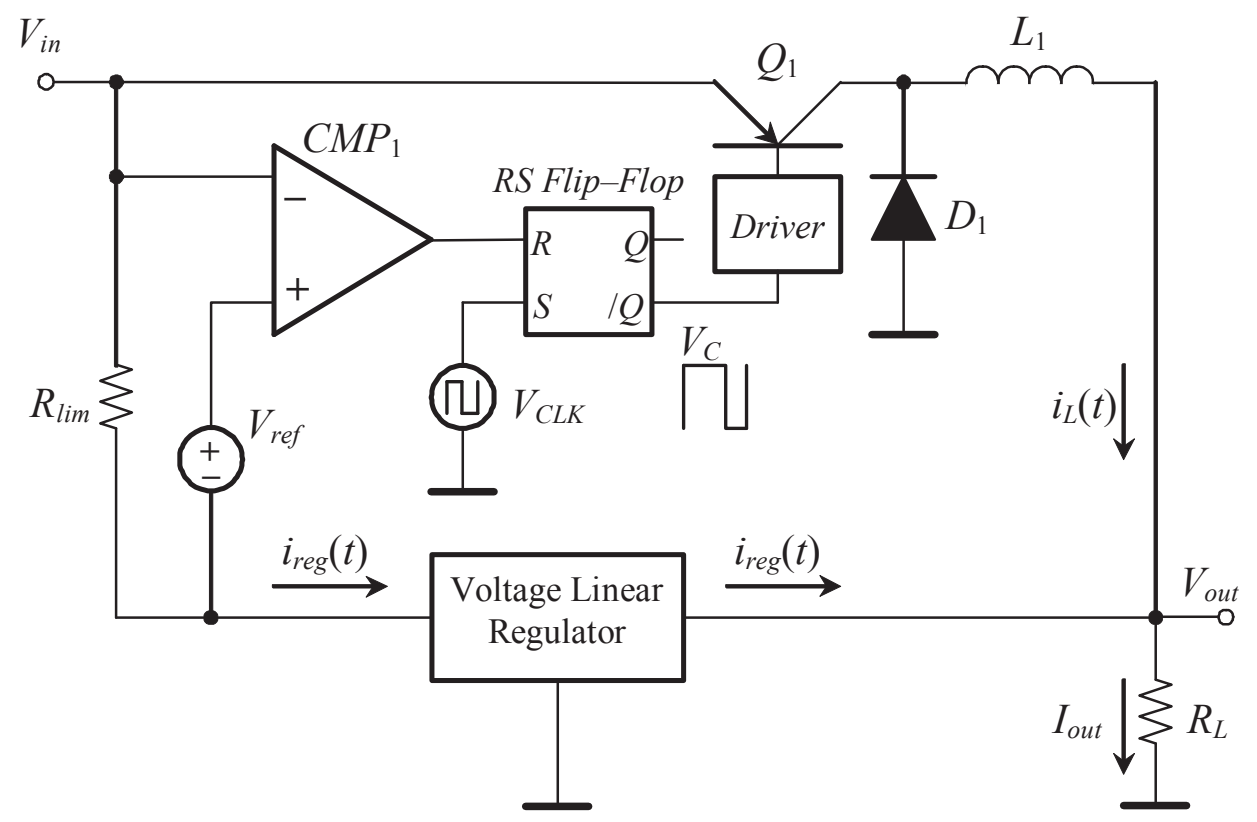

Fig. 4. Schematic of the constant-frequency linear-assisted converter that has been used to validate the proposed structure. 
Figure 5 shows the waveforms of the constant-frequency linear-assisted converter with $V_{i n}=15 \mathrm{~V}$. In the same figure, it is observed the response to an input voltage step from $15 \mathrm{~V}$ to 18 $V$ in the time instant $t=400 \mu s$, and a step change of the load resistor of the $50 \%$ (also from $10 \Omega$ to $5 \Omega$ ) in $t=800 \mu \mathrm{s}$. The output voltage is fixed, thanks to the linear regulator, to $7 \mathrm{~V}$ and the current $I_{\gamma}$ has been adjusted in this case to $100 \mathrm{~mA}$.

The clock signal determines the start of $T_{O N}$ interval and the output of the comparator determines the beginning of $T_{O F F}$.

The current sensing of the linear regulator acts as a control loop and establishes the switch duty ratio in a similar way that the well-known PWM modulation. Figure 6 shows in detail the waveforms of the converter in the steady state.

Actually, note that in this converter the control is a currentmode control. With regard to the classical current-mode control, in this converter variations of the inductor current are measured indirectly thanks to the measure of the variations in the linear regulator.

As it is well known, the current-mode control has an important limitation: for switch duty ratios greater than 0.5 the system is unstable [5]. To prevent this limitation in the currentmode control, a slope compensation has to be added to the control loop in order to provide stability, to prevent subharmonic oscillations, and to provide a feedforward property.

Figure 7 shows the constant-frequency linear-assisted converter with a system that implements this slope compensation by means of an integrator with reset.

\section{CONCLUSIONS}

This paper shows the proposal of a linear-assisted converter or linear-\&-switching hybrid converter with constant switching frequency. With regard to the linearassisted converters with variable switching frequency, the presented structure eliminates the disadvantages derived from having variable switching frequency.

The introduced control loop is based on the current-mode technique that has as a main drawback, as usual in DC-DC converters, the instability of the loop when switch duty ratios are greater than 0.5 . This work has introduced the technique based on slope compensation in order to make stable the linear-assisted converter. The proposed modifications guarantee the stability and make possible this new mode of work in this kind of converters.

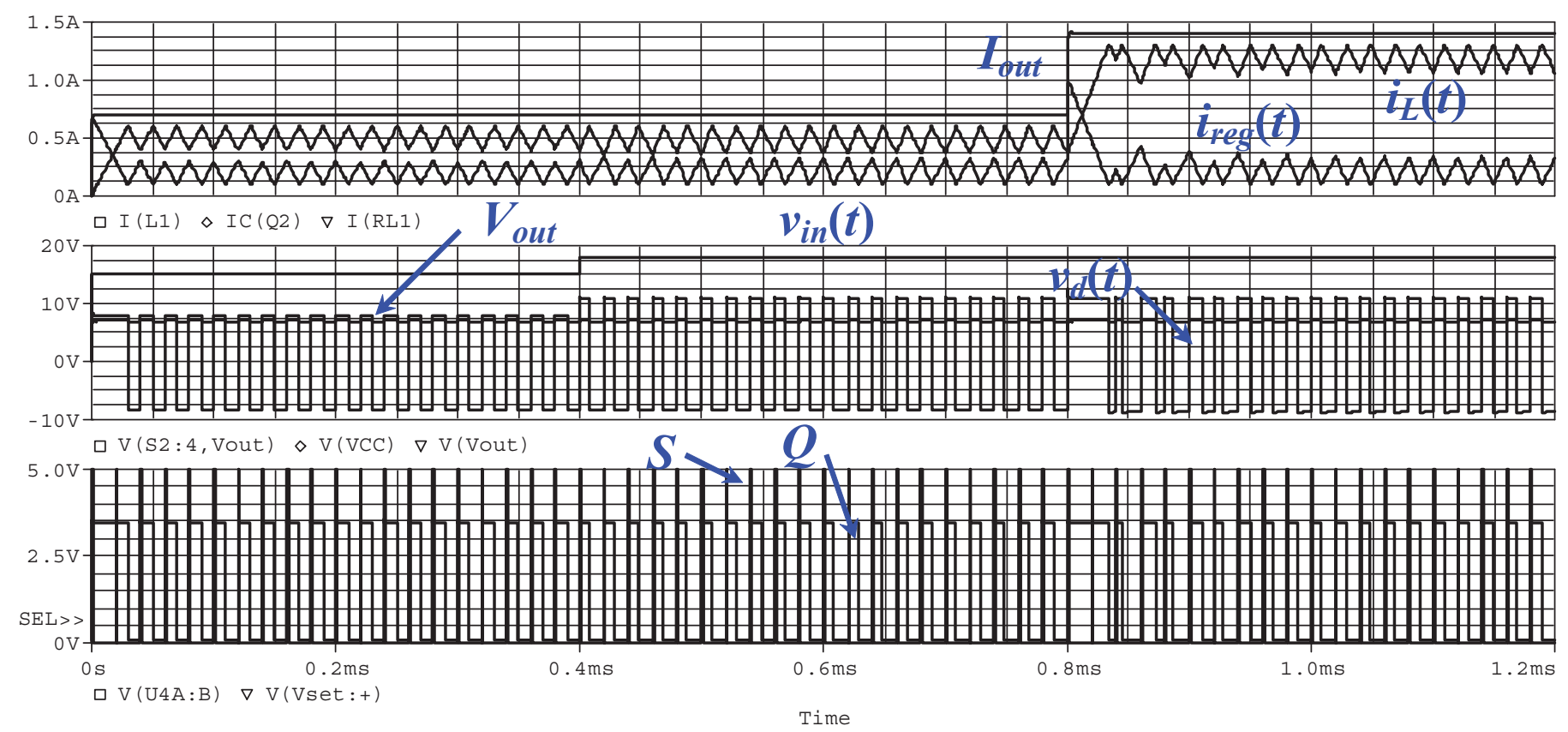

Fig. 5. Transient response of the constant-frequency linear-assisted converter with $V_{i n}=15 V$. It can be seen the response of the circuit to an input step from $15 V$ to $18 \mathrm{~V}$ in the time instant $t=400 \mu \mathrm{s}$, and when there is a decrement of the load resistance from $10 \Omega$ to $5 \Omega$ in $t=800 \mu s$. 


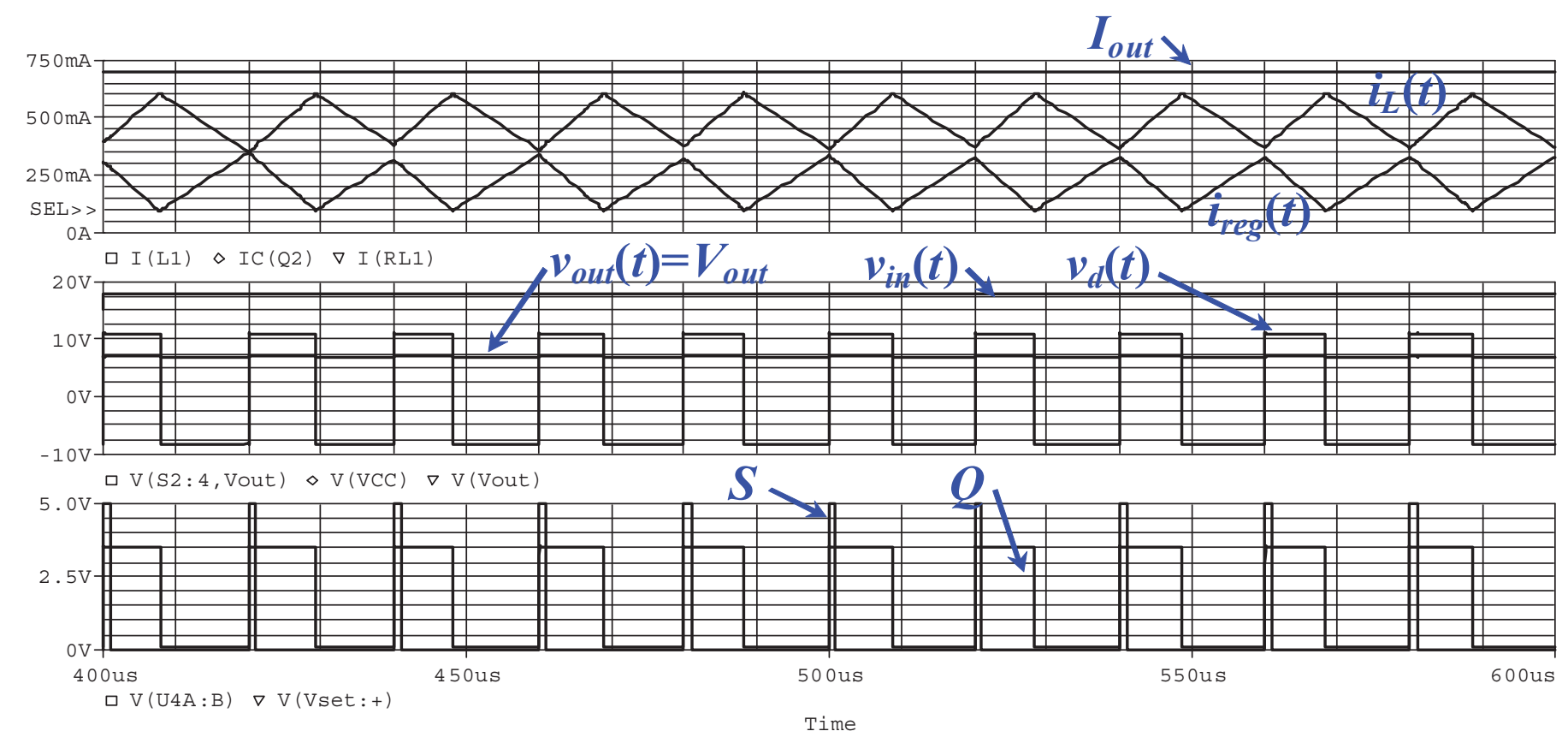

Fig. 6. Detail of the waveforms of the constant-frequency linear-assisted converter in the steady state.

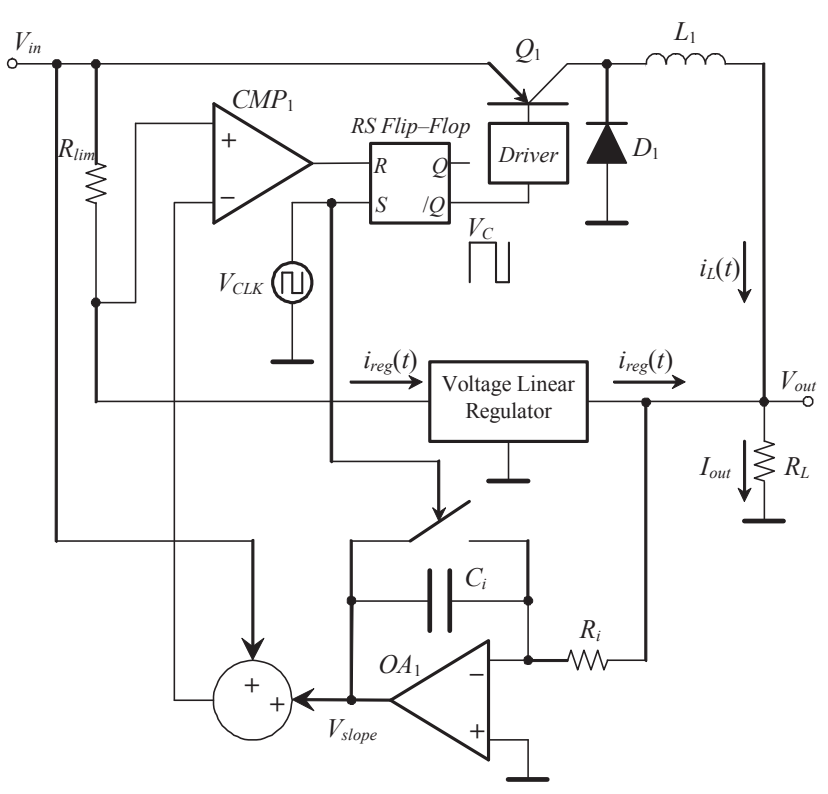

Fig. 7. Schematic of the constant-frequency linear-assisted converter with the implementation of the slope compensation.

\section{ACKNOWLEDGMENT}

This work has been partially supported by the Spanish Ministerio de Economía y Competitividad by Projects DPI2013-42458-P, and DPI2013-47799-C2-2-R.

\section{REFERENCES}

[1] R. K. Dokania, and G. A. Rincón-Mora. "Cancellation of Load Regulation in Low Drop-Out Regulators". Electronic Letters, vol. 38 $\left(n^{\circ} 22\right):$ pp. 1300-1302, $24^{\text {th }}$ October, (2002)

[2] V. Grupta, G. A. Rincón-Mora, and P. Raha "Analysis and Design of Monolithic, High PSR, Linear Regulator for SoC Applications". Proceedings of the IEEE International SoC Conference: pp. 311-315, (2004).

[3] R. W. Erickson, and D. Maksimovic. "Fundamentals of Power Electronics". $2^{\text {nd }}$ edition, Ed. Kluwer Academic Publishers, (2001).

[4] J. G. Kassakian, M. F. Schlecht, and G. C. Verghese. "Principles of Power Electronics". Ed. Addison-Wesley, (1991).

[5] N. Mohan, T. M. Underland, and W. P. Robbins. "Power Electronics: Converters, Applications and Design". $3^{\text {rd }}$ edition. Ed. John Wiley \& Sons, (2003).

[6] G. Villar, E. Alarcón, F. Guinjoan, and A. Poveda. "EfficiencyOriented Switching Frequency Tuning for a Buck Switching Power Converter". IEEE International Symposium on Circuits and Systems (ISCAS'05), (2005).

[7] X. Zhou, P. L. Wong, P. Xu, F. C. Lee, and A. Q. Huang. "Investigation of Candidate VRM Topologies for Future Microprocessors". IEEE Transactions on Power Electronics, vol. 15 (nº): pp. 1172-1182, (November 2000).

[8] H. Ertl, J. W. Kolar, and F. C. Zach. "Basic Considerations and Topologies of Switched-Mode Assisted Linear Power Amplifiers". IEEE Transactions on Industrial Electronics, vol. 44 ( $\left.{ }^{\circ} 1\right)$ : pp. 116123, (February 1997).

[9] H. Martínez, A. Conesa. "Modeling of Linear-Assisted DC-DC Converters". European Conference on Circuit Theory and Design 2007 (ECCTD 2007), $\left(27^{\text {th }}-30^{\text {th }}\right.$ August 2007).

[10] A. Conesa, H. Martínez, J. M. Huerta. "Dynamic Analysis of Hybrid DC-DC Converters". 12 th European Conference on Power Electronics and Applications (EPE 2007), $\left(2^{\text {nd }}-5^{\text {th }}\right.$ September 2007). 